\title{
Das Institut für Fremdenverkehr und Verkehrswirtschaft an der Hochschule St. Gallen
}

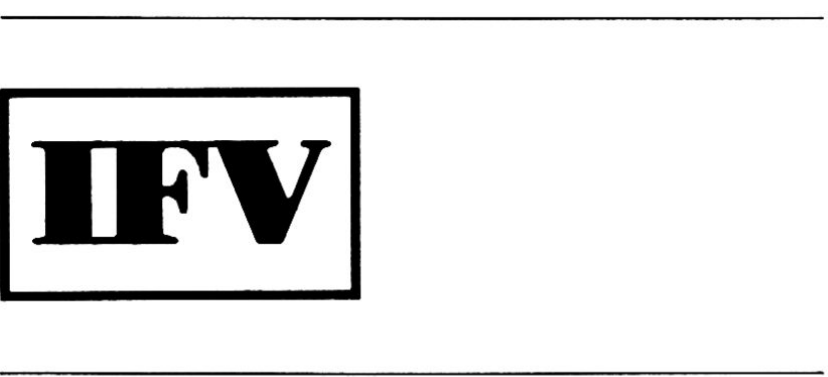

\section{Gründung und Zielsetzung}

Bereits 1941 ist das Seminar für Fremdenverkehr und Verkehrspolitik an der damaligen Handelshochschule gegründet worden, um die kurz zuvor eingeführten Vorlesungen zum Fremdenverkehr und zur Verkehrspolitik zu unterstützen. Die beiden ersten Direktoren, Prof. Dr. Walter Hunziker und Prof. Dr. Walter Fischer, vermochten dem Seminar recht bald nationale Bedeutung zu geben, zumal das Seminar durch Organisation von Weiterbildungskursen und durch Publikation einschlägiger Untersuchungen eine wichtige Aufgabe erfüllte. Mit dem Eintritt von Prof. Dr. Claude Kaspar in die Institutstätigkeit erfuhr das Institut eine Erweiterung des Aufgabenbereiches, indem die Grundlagen- und die Auftragsforschung immer mehr zum Schwerpunkt des Instituts wurden.

Gemäß Institutssatzung vom 28. Mai 1971 bestehen die wichtigsten Aufgaben in der

- «wissenschaftlichen Bearbeitung von verkehrs- und volkswirtschaftlichen Problemen des Fremdenverkehrs und der Verkehrswirtschaft;

- Förderung der Ausbildung eines mit wissenschaftlichem Rüstzeug versehenen Nachwuchses für leitende Stellungen im Fremdenverkehr und in der Verkehrswirtschaft im Rahmen der Lehrtätigkeit an der Hochschule St. Gallen;

- Weiterbildung der höhern und mittleren Kader auf den vom Institut betreuten Gebieten durch Vorträge, Kurse und sonstige Veranstaltungen;

- Sammlung der für Forschungs- und Lehrtätigkeit erforderlichen Dokumentation und Spezialliteraturn.

\section{Organisation}

Das Institut wird organisatorisch von einem $\mathrm{Ge}$ schäftsleitenden Ausschuß (Vorsitz Prof. Dr. A. Nydegger) sowie von der Direktion (Prof. Dr. C. Kaspar und Dr. H.P.Schmidhauser) getragen. Eine wichtige ideelle und finanzielle Aufgabe erfüllt die "Gesellschaft zur Förderung des Instituts für Fremdenverkehr und Verkehrswirtschaft». Unter der aktiven Leitung von Dr.M.Redli, a.Präsident GD PTT, vereinigt die Gesellschaft rund 150 Organisationen, Unternehmungen und Einzelpersonen aus der Fremdenverkehrs- und Verkehrswirtschaft. Dadurch kann die Beziehung von Wissenschaft und Praxis besonders eng gestaltet werden, zum großen Vorteil beider Seiten. Eine seit mehreren Jahren bestehende «Vereinigung ehemaliger Studierender der Richtungen Fremdenverkehr und Verkehrswirtschaft der Hochschule St.Gallen» stellt die Kontakte zu den Absolventen sicher und ermöglicht einen besseren Einsatz der Ehemaligen durch eine vom Institut geführte Stellenvermittlung.

\section{Forschungstätigkeit}

Die Förderung der Forschung auf den beiden Lehrgebieten des Fremdenverkehrs und der Verkehrswirtschaft entspricht zweifellos einem Bedürfnis. Zahlreich sind auch die Aufträge der Praxis, die verschiedensten betriebs- und volkswirtschaftlich orientierten Probleme zu untersuchen und Lösungsvorschläge auszuarbeiten. Einen über die Landesgrenzen hinausreichenden Namen hat sich das Institut bei der Erarbeitung einer wissenschaftlich fundierten Fremdenverkehrs- und Verkehrswirtschaftslehre erworben. Entsprechende Lehrbücher sind zu Standardwerken geworden. Einen wichtigen Schwerpunkt bilden die unter der Leitung von Dr. H. P. Schmidhauser stehenden Repräsentativerhebungen über den schweizerischen Reisemarkt. Verschiedene Studien sind in letzter Zeit im Auftrag des Bundes (Wochenendausflugsverkehr im Zusammenhang mit der Gesamtverkehrskonzeption, Ermittlung regionalwirtschaftlicher 
Auswirkungen des Baus bzw. Nichtbaus von Autobahnen im Rahmen der Überprüfung von Nationalstraßenstrecken, Problematik der Zweitwohnungen bzw. der Parahotellerie usw.) durchgeführt worden. Der Forschungstätigkeit und immer mehr der Studentenausbildung dient die hauptsächlich mit Zeitschriften und Fachliteratur dotierte Handbibliothek des Instituts.

\section{Lehrtätigkeit}

Durch die Doppeltätigkeit der beiden Direktoren, Prof. Dr. Kaspar und Dozent Dr. Schmidhauser, im Institut und als Dozenten an der Hochschule St. Gallen für Wirtschafts- und Sozialwissenschaften wird die wichtige Einheit von Lehre und Forschung sichergestellt. Die Vertiefungsrichtung Fremdenverkehr und Verkehrswirtschaft ist Teil der wirtschaftswissenschaftlichen Ausbildung. Dadurch können Diplomarbeiten und Dissertationen aus dem Themenbereich Fremdenverkehr und Verkehrswirtschaft ausgearbeitet werden.

\section{Kurstätigkeit}

Nach wie vor ist die Vortragstätigkeit der Institutsmitglieder sehr rege, womit ein wertvoller Gedankenaustausch mit der Praxis gepflegt werden kann. Die alljährlich durchgeführten Kursveranstaltungen des Instituts setzen sich zum Ziel, aktuelle Fragen der betreuten Gebiete zur Diskussion zu stellen, um hiefür gültige Lösungen zu suchen. Die Arbeitstagungen der Ehemaligen ermöglichen einen Dialog zwischen dem Institut und den Ehemaligen und sind im weitern als Weiterbildungsseminare konzipiert.

\section{Veröffentlichungen}

Die Reihe «St. Galler Beiträge zum Fremdenverkehr und zur Verkehrswirtschaft» möchte einem weitern Kreis von Interessenten Arbeiten des Instituts und
Untersuchungen seiner Mitarbeiter sowie Dissertationen aus dem Gebiet des Fremdenverkehrs und der Verkehrswirtschaft zugänglich machen. Die Schriftenreihe wird ergänzt durch eine Instituts-Sonderreihe für ausgezeichnete Diplomarbeiten sowie durch periodisch erscheinende "Instituts-Mitteilungen». Zusammen mit dem Berner Forschungsinstitut für Fremdenverkehr gibt zudem das Institut die international anerkannte "Zeitschrift für Fremdenverkehr» heraus. Das Institut publiziert neu ein Jahrbuch der schweizerischen Verkehrswirtschaft.

\section{Ausblick}

Die finanzielle Regelung der St. Galler Institute zwingt diese, der Auftragsforschung eine gewisse Priorität einzuräumen. Dadurch wird die Grundlagenforschung etwas eingeengt, obwohl letztere durch Forschungsbeiträge vielfach gefördert wird. Die Auftragsforschung besitzt indessen den Vorteil, daß sich das Institut eingehend mit den Problemen der Fremdenverkehrs- und Verkehrspraxis auseinandersetzen muß. Dies kommt nicht zuletzt der problemorientierten Lehre an der Hochschule St. Gallen zugute. Den internationalen und den nationalen Kontakten wird auch in Zukunft besondere Aufmerksamkeit geschenkt werden, zumal die Beziehungen zur Fremdenverkehrs- und Verkehrspraxis sowie zu Behörden und $\mathrm{zu}$ andern wissenschaftlichen Institutionen und Gremien für die erfolgreiche Erfüllung der Institutsaufgaben von entscheidender Bedeutung sind.

\section{Kaspar}

Institut für Fremdenverkehr und Verkehrswirtschaft an der Hochschule St. Gallen Varnbüelstr. 19, 9000 St. Gallen, Tel. 071/22 8755 